\title{
Analysis of metabolic pathways in mycobacteria to aid drug-target identification
}

Bridget P. Bannerman ${ }^{1}$, Sundeep C. Vedithi ${ }^{1 \pi}$, Jorge Júlvez ${ }^{1,2 \pi}$, Pedro Torres $^{1 \pi}$, Vaishali $P$. Waman $^{1 \&}$, Asma Munir ${ }^{1 \&}$, Vitor Mendes ${ }^{1 \&}$, Sony Malhotra ${ }^{3 \&}$, Marcin J. Skwark ${ }^{1 \&}$, Stephen G. Oliver $^{1}$ and Tom L. Blundell ${ }^{1 * \pi}$

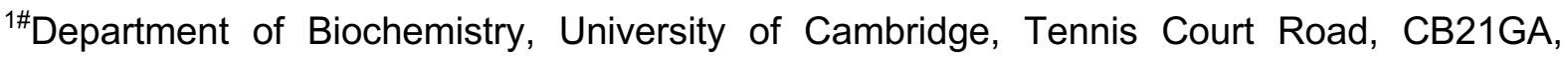
Cambridge, UK.

${ }^{2}$ Department of Computer Science and Systems Engineering, University of Zaragoza, María de Luna 3, 50018 Zaragoza, Spain

${ }^{3}$ Department of Biological Sciences, Birkbeck College, University of London, London, UK

* Corresponding authors

E-mail: tom@cryst.bioc.cam.ac.uk and bee1bee22@gmail.com

TThese authors contributed equally to this work.

\&These authors also contributed equally to this work.

Full Title: Comparative analyses of metabolic pathways in mycobacteria: Insights into the identification and characterisation of new drug targets in M. tuberculosis, M. leprae and $M$. abscessus 


\section{Abstract}

Three related mycobacteria are the cause of widespread infections in man and are the focus of intense research and drug-discovery efforts in the face of growing antimicrobial resistance. Mycobacterium tuberculosis, the causative agent of tuberculosis, is currently one of the top ten causes of death in the world according to WHO; $M$. abscessus, a group of non-tuberculous mycobacteria causes lung infections and other opportunistic infections in humans; and $M$. leprae, the causative agent of leprosy, remains endemic in tropical countries. There is an urgent need to design alternatives to conventional treatment strategies, due to the increase in resistance to standard antibacterials. In this study, we present a comparative analysis of chokepoint and essentiality datasets that will provide insight into the development of new treatment regimes. We illustrate the key metabolic pathways shared between these three organisms and identify drug targets with a wide metabolic impact that are common to the three species. We demonstrate that $72 \%$ of the chokepoint enzymes are proteins essential to Mycobacterium tuberculosis. We show also that $78 \%$ of the drug targets, prioritized based on their presence in multiple paths on the metabolic network, are present in pathways shared by M. tuberculosis, M. leprae and $M$. abscessus, including biosynthesis of amino acids, carbohydrates, cell structures, fatty acid and lipid biosynthesis. A further $17 \%$ is found in the prioritised pathways shared between $M$. tuberculosis and $M$. abscessus. We have performed comparative structure modelling of potential drug targets identified using our analysis in order to assess druggability and demonstrate the importance of chokepoint analysis in terms of drug target identification.

\section{AUTHOR SUMMARY}

Computer simulation studies to design new drugs against mycobacteria 


\section{Introduction}

Pathogenic mycobacteria, such as M. tuberculosis, the causative agent of tuberculosis in humans, and $M$. leprae, the causative agent of leprosy, have developed complex metabolic features that enable them to explore their hosts' environments and compete with the host for nutrients. While $M$. leprae evolved by substantially reducing the size of its genome, losing many metabolic pathways and undergoing further pseudogenization (1,2), M. tuberculosis retained metabolic redundancy and an ability to use many different types of sources for nitrogen, carbon and energy, such as sugars, host-derived fatty acids, glucose, tricarboxylic acids, amino acids and cholesterol (3). However, during infection it mostly relies on hostderived fatty acids as the primary energy source (3). M. abscessus, a mycobacterium that causes opportunistic infections in cystic fibrosis patients, has developed numerous evolutionary metabolic adaptations (4-6).

Metabolism refers to the chemical reactions organised into pathways and occurring in the cells of all living organisms. These pathways are broadly classified in two main categories: anabolic and catabolic, and are specialised to enable cell survival and replication. Comparative analyses of metabolism in mycobacteria can highlight and identify unique metabolic features, which will be useful in disease management and drug target identification.

Catabolic pathways are energy-producing processes whereby nutrients are broken down into smaller molecules; typical examples are glycolysis, the Krebs cycle, electron transport chain, and fermentation (3,7-10). These are ancient pathways, widely conserved in most organisms, including mycobacteria. Glycolysis and the Krebs cycle, in addition to other energy-producing processes, such as the glyoxylate, pentose phosphate pathway and the electron transport chain, are among the core energy metabolic pathways in M. tuberculosis, M. abscessus and M. leprae $(11,12)$.

Anabolic pathways are biosynthetic processes comprising the energy-consuming reactions that generate the biomass of mycobacterial cells by synthesizing cellular components such as lipids, carbohydrates, amino acids, and nucleic acids $(7,8)$. These pathways are specialised in mycobacteria and enable them to maintain their structural integrity amidst harsh cellular conditions such as depletion of nutrients and responses of the host immune system during 
infection. Examples of conserved anabolic pathways across mycobacterial species are the amino acid and nucleic acid biosynthetic pathways. Of particular note is the complex arginine pathway, with a variety of anabolic arginine genes in $M$. tuberculosis (13), the lactic acid bacteria (14) and in Pseudomonas (15). L-arginine also occurs as a source of both carbon and nitrogen in bacteria and other microorganisms (15) and the biosynthetic pathway of Larginine has been confirmed as essential to M. tuberculosis (16).

Enzymes of the arginine biosynthetic pathway, including amino-acid acetyltransferase (ArgA) and $\mathrm{N}$-acetyl-gamma-glutamyl-phosphate reductase $(\mathrm{ArgC})$ in $M$. tuberculosis, respectively encoded by the genes, Rv2747 and Rv1652, have previously been identified as high confidence drug targets $(17,18)$. ArgA catalyses the first of eight enzymatic steps in the biosynthesis of arginine from glutamate as well as being involved in the synthesis of ornithine and proline. This involves the activation and reduction of the gamma-carboxyl group in glutamate to produce glutamate-5-semialdehyde. The subsequent steps, catalysed by acetylglutamate kinase (ArgB) and ArgC, involve the phosphorylation of $\mathrm{N}$-acetyl-L-glutamate to $\mathrm{N}$-acetyl-L-glutamate 5-phosphate and its subsequent reduction to N-Acetyl-L-glutamyl 5phosphate. Acetylornithine aminotransferase (ArgD) catalyses the reaction from N2-acetyl-Lornithine to $\mathrm{N}$-acetyl-L-glutamate 5-semialdehyde (13).

During the second stage of arginine biosynthesis, ornithine carbamoyltransferase (ArgF) catalyses the formation of citrulline from carbamoyl phosphate, which is in turn catalysed to ( $\mathrm{N}$ (omega)-L-arginino) succinate by argininosuccinate synthase (ArgG). L-arginine is produced from 2-(N(omega)-L-arginino) succinate in a reaction catalysed by argininosuccinate lyase $(\mathrm{ArgH})$. The intermediate compound, carbamoylphosphate, also provides an alternative to the second stages of L-arginine biosynthesis via the ornithine biosynthetic pathway. Both arginine and ornithine occur as precursors in diverse arginine degradative pathways and in the biosynthesis of polyamines (19-21). Carbamoyl phosphate is also an intermediate in the synthesis of pyrimidines that are the activated precursors of DNA and RNA and a requirement for cell growth (15).

A comprehensive understanding of the enzymes of essential metabolic pathways in mycobacteria, such as the arginine biosynthetic pathway, is necessary for novel drug discovery and elucidating the role of mutations in drug resistance. Currently, crystal structures of some of the enzymes of the arginine pathway of $M$. tuberculosis H37RV exist in the Protein Data Bank (22), including ArgA (5YGE) (23), ArgB (2AP9), ArgC (2nqt, 2i3g, 2i3a) (18) ArgF (2P2G, 2I6U) (18) and ArgJ (3IT4, 3IT6) (24,25) However, experimental structures of ArgD, ArgG and ArgH are lacking for M. tuberculosis, M. abscessus and M. leprae. 
To understand the metabolism of an organism at a system level, it is necessary to identify and reassemble its constitutive parts (metabolites, reactions and uptake processes) which can then be integrated in genomic-scale metabolic networks that taken together can simulate the normal function of a cell (26). These metabolic reconstructions have been used in many different ways from identifying genes essential for growth and thus establishing new drug targets to modelling quantitative drug-dose responses $(27,28)$. A complementary approach to predicting drug targets for mycobacteria is to elucidate the enzymes catalysing reactions that are the sole producers of a specific metabolite or the sole consumers of that metabolite, by querying the annotated genome of an organism, a process referred to as chokepoint analysis (29). Chokepoint enzymes of mycobacterial genomes are critical to bacterial survival and represent potential drug targets from the metabolic networks of the bacteria.

In this study, we show results from comparative pathway and chokepoint analyses of $M$. tuberculosis, $M$. abscessus and $M$. leprae. We review essential pathways common in the three species and predict potential drug targets for infectious diseases such as leprosy and tuberculosis. We focus on the arginine biosynthetic pathway. For this, we describe comparative modelling procedures to predict the three-dimensional structures of selected enzymes that lack experimental structures, as well as druggability analyses of potential drug targets. We show how the identification and combination of chokepoint reactions within the metabolic networks can indicate essentiality vital for survival of the pathogen. We show how bringing chokepoint analysis of the reactions together with the ligand-binding ability of the enzymes catalysing them is central to the selection of targets for drug discovery.

\section{Results}

\section{Pathway analyses}

For a comparative analysis of pathways shared between $M$. tuberculosis H37Rv, $M$. abscessus and $M$. leprae, we used the newly reconstructed $M$. tuberculosis model iEKVIII (30) and the draft BioCyc pathway databases of M. abscessus and M. leprae. There were 297 pathways in M. abscessus, 212 pathways in M. tuberculosis and 135 pathways in M. leprae (S1 Table). The larger number of metabolic pathways in $M$. abscessus is consistent with the fact that it is an opportunistic pathogen with a larger genome size of 5MB (31) compared to the relatively smaller genome sizes of the obligate pathogens $M$. tuberculosis (4MB) and $M$. leprae (3.3 MB). There are fewer metabolic pathways in $M$. leprae as it has undergone significant genomic reduction with a small genome size of $3.3 \mathrm{MB}$ and has relatively poor genome annotation (32). 
We found 105 pathways in common between the three mycobacteria (M. tuberculosis, $M$. abscessus and $M$. leprae), 152 pathways shared between $M$. tuberculosis and $M$. leprae and 112 pathways shared between $M$. abscessus and $M$. leprae. The differences in the numbers of shared pathways are mainly due to differences in the number of biosynthetic and/or degradative pathways of these organisms. The loss of genes in $M$. leprae due to reductive evolution has resulted in fewer metabolic pathways. The species-specific metabolic pathways identified in M. tuberculosis include processes involved in cellulose binding, hydrolase activity, as well as carbohydrate and polysaccharide binding. In the case of $M$. abscessus, speciesspecific biological processes include an increase in the number of reactions concerned with the biosynthesis of polyamines, thiols and L-citrulline, as well as the number of membrane transport proteins. We analysed these pathways further to identify drug targets common to the three species using the chokepoint analysis algorithm (Fig 1; S1 File).

\section{Chokepoint analysis for the identification of drug targets}

The concept of 'chokepoint reactions' was introduced by Yeh and colleagues in 2004 (29), when they demonstrated how the metabolic network of an organism can be queried to predict potential drug targets. A chokepoint reaction of a metabolic network must not only be a reaction that is either the only consumer or the only producer of a given metabolite, but in addition, it is required that such a metabolite is not a dead-end metabolite, i.e. each chokepoint reaction should be balanced by at least one other reaction that produces or consumes that metabolite.

Chokepoint reactions are believed to be essential to the bacteria and are therefore potential drug targets. We initially analysed the three metabolic pathway databases of $M$. tuberculosis, $M$. abscessus and $M$. leprae for the unique reactions within the metabolic networks using the chokepoint algorithm. We excluded the chokepoint reactions found in humans and further restricted our selection to chokepoint enzymes that are present in more than one pathway in order to assess which enzymes will produce a greater impact on the metabolic network (Fig 2; S2 Table).

Of the 333 potential drug targets initially identified in M. tuberculosis, 24 targets selected for further analyses were found to be involved in more than one pathway; these include lipid metabolism, coenzyme transport and metabolism, energy production and conversion, amino acid and nucleotide transport and metabolism (Table A in S2 Table). We postulate that potential inhibitors of targets identified in multiple pathways would have a more significant impact on the metabolic network of the bacterium than the inhibitors of a single pathway. In 
M. abscessus, 313 potential drug targets were initially identified and 15 were involved in more than one pathway, including cell wall and cell processes, coenzyme transport and metabolism, energy production and conversion, amino acid and nucleotide transport and metabolism; these targets were selected for further analyses (Table B in S2 Table). Similarly, 20 potential targets of the 183 identified in $M$. leprae were involved in more than one pathway including lipid transport and metabolism, nucleotide transport and metabolism, cell wall/membrane/envelope transport and metabolism/amino acid transport and metabolism / coenzyme transport and metabolism and these were selected for further analyses (Table $\mathrm{C}$ in S2 Table).

Based on in vivo and in vitro essentiality patterns, we confirmed that $75 \%$ of the chokepoint enzymes are essential proteins to Mycobacterium tuberculosis. $(33,34)$. Among the prioritized drug targets, $78 \%$ of the chokepoint enzymes are present in pathways shared between $M$. tuberculosis, M. leprae and $M$. abscessus; these include the biosynthesis of amino acids, such as arginine and ornithine, nucleotide transport and metabolism and pyrimidine biosynthesis (Tables A-C in S2 Tables). Here we focus on the arginine biosynthetic pathway in the $M$. tuberculosis network.

We performed Flux Balance Analysis (FBA) (35) on this pathway to assess the essentiality of $\operatorname{ArgD}, \mathrm{ArgH}$ and other enzymes in the M. tuberculosis network. We implemented both FBA and Flux Variability Analysis (FVA) on the m7H10 medium (a growth medium of choice for cultivating $\mathrm{M}$ tb) using the 'biomass' reaction as the objective function and knocking out $\mathrm{Arg} \mathrm{D}$ and $\mathrm{ArgH}$ to assess the impact on the metabolic network and to compute the minimum and maximum fluxes that the reactions can take. The maximum growth rates $\left(h^{-1}\right)$ predicted by FBA after knocking out $\mathrm{ArgB}, \mathrm{ArgC}, \mathrm{ArgD}$ and $\mathrm{ArgH}$ are reported in S3 Table.

A maximum growth rate of zero confirms the in-silico essentiality of the target enzymes ArgD and $\mathrm{ArgH}$. Moreover, FVA (35) demonstrates a zero-flux distribution of the reactions when either of these essential enzymes is absent (Fig 3) in M. tuberculosis. Our FVA results also show that $\mathrm{ArgF}, \mathrm{ArgG}$ and ArgJ exhibit zero flux distribution of reactions when they are absent from the pathway, although the growth rate of the bacterium when ArgA is knocked out is the same as the wild type. Therefore, we propose that $\operatorname{ArgB}, \operatorname{ArgC}, \operatorname{ArgD}, \mathrm{ArgF}, \mathrm{ArgG}, \mathrm{ArgH}$ and ArgJ represent critical enzymes for the continued growth and viability of $M$. tuberculosis but $\operatorname{Arg} \mathrm{A}$ is not essential to the bacterium.

\section{Comparative 3D modelling}


We identified structures for $\mathrm{ArgB}, \mathrm{ArgC}$ and $\mathrm{ArgF}$, chokepoint enzymes present in $M$. tuberculosis, $M$. abscessus, and M. leprae that are present in the Protein Data Bank (PDB); these are listed on Tables A-C in S2 Tables. Comparative modelling techniques $(36,37)$ were therefore employed to predict the structures for $\mathrm{ArgD}$ and $\mathrm{ArgH}$ that are not currently available in the PDB.

ArgD: The ArgD (acetylornithine aminotransferase) protomer of $M$. tuberculosis was modelled using the templates 1vef (acetylornithine aminotransferase from Thermus thermophiles) with an identity of $45 \%$ and a coverage of $92 \%$ and $4 a d b$ (succinyl-ornithine transaminase from $E$. coli with an identity of $42 \%$ and a coverage of $96 \%$ ). The overall Molprobity score of the model is 1.36 at the 98th percentile $(38,39)$. Structural superimposition of the model with each of the templates $4 \mathrm{adb}$ and 1vef resulted in a root mean square deviation (RMSD) of $0.203 \AA$ and $0.908 \AA$, respectively. A homodimer was then built by superimposing the protomers with each chain of the template 1vef; we have run Steepest Descent, Conjugate gradient and Modrefiner $(40-42)$ to reduce steric clashes and to improve the quality of the models (Fig 4A in Fig 4).

ArgH: The ArgH (argininosuccinate lyase) protomer was modelled using the templates 2e9f (argininosuccinate lyase from Thermus thermophilus (Identity $=45 \%$ and coverage $=95 \%$ )) and $1 \mathrm{k} 62$ (argininosuccinate lyase from human (Identity $=43 \%$ and coverage $=97 \%)$ ). The resulting model has an overall Molprobity score of 1.44 at 96th percentile $(38,39)$. Structural superimposition with the templates $1 \mathrm{k} 62$ gave RMSD of $0.577 \AA$ and $1.069 \AA$ with $2 \mathrm{e} 9 \mathrm{f}$. A homotetramer was built by superimposing the protomer with each chain of the template 2e9f and we improved the quality of the models by running Steepest Descent, Conjugate gradient and Modrefiner (40-42) (Fig 5A in Fig 5).

In ArgD, the pyridoxal 5'-phosphate (PLP), the co-factor in the transamination reaction binding site, is located close to the dimeric interface (Fig 4B in Fig 4). It is located in the active centre cleft and forms multiple interatomic interactions with the surrounding residues. The oxygen atom of the phosphate moiety of PLP forms hydrogen-bond interactions with the main chain nitrogen atom of Thr282 (Fig 4C in Fig 4). It also forms weak polar interactions with side chain atoms of Thr282 and Val226. The pyridine ring forms multiple proximal $\pi$ interactions with other the aromatic residues in the binding site. It forms a face to edge $\pi$ interaction with Phe139 and carbon- $\pi$ interaction with Val226. The Fragment Hotspots Maps which specifically highlight fragment-binding sites and their corresponding pharmacophores (43), overlapped the binding site corresponding to hydrophobic, donor and acceptor regions with a score of 17 . ArgH, the L-arginine binding site is located at the interface between domains one 
and two (Fig 5B in Fig 5). Arginine interacts with the surrounding residues by the formation of a hydrogen bond with Asn111 and m interactions with Tyr320 (Fig 5C in Fig 5).

\section{Sequence-structure comparisons between human and Mtb ArgD and ArgH:}

A position-specific iterated BLAST (PSI-BLAST) search against the PDB of Mtb ArgD on the human proteome revealed six structural hits with sequence identities ranging from $30-36 \%$ (S4 Table). Alignment of Mtb ArgD sequence with the structure of 2BYJ (having the highest identity with the sequence) using JOY (44), revealed conserved residues across the PLP binding site between both the proteins. The alignments are depicted in Supp Fig 1A. Structural superimposition of chain $A$ of $A r g D$ model with chain $A$ of $2 B Y J$ resulted in an RMSD of $1.806 \AA$ (Supp Fig 1B).

PSI-BLAST of Mtb ArgH with the human proteome resulted in five hits on the PDB database with sequence identity ranging from $24-43 \%$ (S5 Table). L-Arginine in Mtb ArgH forms two hydrogen bonds, one with the sidechain oxygen atom of Asn111 and the other with sidechain nitrogen of the Lys328. It also forms hydrophobic contacts with Val114, Arg110 and Tyr320. JOY alignment (44) was performed for $\mathrm{Mtb} \mathrm{ArgH}$ and $1 \mathrm{AOS}$ proteins and conserved regions were highlighted (Supp Fig 2A). Structural superimposition of chain A of human ArgD (1AOS) with chain A of Mtb ArgH model resulted in an RMSD of $0.751 \AA$ (Supp Fig 2B). In Mtb ArgD, three Fragment Hotspots were mapped in the binding sites for PLP, N-Acetyl-glutamate semialdehyde and glutamate (Supp Fig 3A) while in 2BYJ (Human ArgD), only one hotspot was mapped (Supp Fig 3B) at the PLP binding site that partially overlaps with that of the Mtb ArgD. Furthermore, in $\mathrm{Mtb} \mathrm{ArgH}$, three hotspots were mapped at the subunit interfaces (Supp Fig 4A). The maps were contoured at the score of 17 indicating higher likelihood for fragment binding. Conversely, no hotspots were mapped on the human $\mathrm{ArgH}(1 \mathrm{AOS})$ at the score of 17 and few apolar regions were noted at a score of 14 (Supp Fig 4B). All the maps were at the subunit interfaces. Mishra and Surolia (45) have recently shown that $\mathrm{ArgH}$ undergoes conformational changes during catalysis that can influence the orientation of the substrate binding sites and subsequently the fragment binding.

The hotspots (43) and cavities (46) for ArgD were used to explore potential binding of two drugs, Amprenavir and Cochicine, to the ArgD protein and the putative interactions were investigated using a molecular docking approach. The structures of both ligands and of the pyridoxal 5' phosphate (PLP) cofactor were retrieved from Drugbank in the simplified molecular-input line-entry system (SMILES) format (47). The SMILES strings were converted to the PDB format and their 3D structures were optimized after adding hydrogens using the 
UFFOptimizeMolecule function of RDKit. The resulting structures were used to generate pdbqt files, using the prepare_ligand.py script provided with MGLTools (48).

The modelled ArgD structure was converted to pdbqt format using MGLTools, and a cubic grid box with 24 A-long edges was manually set to loosely accommodate the PLP binding site. Autodock VINA was used to generate up to 10 poses of each ligand (num_modes=10) within an energy range of $10 \mathrm{kcal} / \mathrm{mol}$ (energy_range=10) and the exhaustiveness was set to 40 . The Open Drug Discovery Toolkit (ODDT) was used to re-score the docked poses, using the RFScore_V3 function, trained on the PdbBind2015 dataset. To increase the robustness of the results, the above-described procedure was repeated 200 times for each ligand and the results were clustered using the "gmx cluster" program, which integrates the GROMACS package (49). The clustering procedure was carried out with a RMSD cut-off of $0.2 \mathrm{~nm}$ and the docking poses were not fitted prior to the clustering, to capture translational and rotational differences. The resulting data were analysed using the DataWarrior software (50), which was also used to generate the scatter-plots and box-plots. The best-scoring pose of the cluster with the highest median score was selected and submitted to the PLIP web-server in order to analyse the formed interactions. The points of interaction of Amprenavir on the hotspot maps of the ArgD proteins on both human and M. tb are shown on Fig 6 and Supp Fig 3c. The PLP binding site identified on the ArgD sequence of $\mathrm{M}$. th is conserved in $M$. leprae and $M$. abscessus; the $M$. tb $\operatorname{ArgD}$ sequence is $83 \%$ identical to that of $M$. leprae and $74 \%$ identical to the $M$. abscessus ArgD sequence (Supp Fig 4). Similarly, the M. tb ArgH sequence is $88 \%$ identical to $M$. leprae and $79 \%$ identical to $M$. abscessus. The conserved domains of $A r g D$ and $A r g H$ indicate similar active and Fragment Hotspots in $M$. leprae and $M$. abscessus as seen in $M$. tuberculosis demonstrating the significance of metabolic pathway analysis in drug target identification of mycobacteria.

\section{Fig Legends}

Fig 1. Pipeline for drug target identification in mycobacteria

Fig 2. Choke point reactions in mycobacteria

Fig 3. Flux Variability Analysis (FVA) to demonstrate the difference in the flux of reactions in the arginine biosynthetic pathway when enzymes, $\operatorname{ArgA}$ and $A r g D$, are absent

Fig 4. Dimeric model of Mtb ArgD with PLP Binding site and interatomic interactions of PLP with the surrounding residue environment

(4A) ArgD homodimer 
4B) The location of the pyridoxal 5'-phosphate (PLP) and the co-factor in the transamination reaction binding site close to the dimeric interface

4C) Formation of hydrogen-bond interactions between the oxygen atom of the phosphate moiety of PLP and the main chain nitrogen atom of Thr282

Fig 5. Tetrameric model of $\mathrm{Mtb}$ ArgH with L-Arginine Binding site and interatomic interactions of L-Arginine with the surrounding residue environment.

5A) $\mathrm{ArgH}$ homotetramer was built by superimposing the protomer with each chain of the template $2 \mathrm{e} 9 \mathrm{f}$

5B) Location of the ArgH L-arginine binding site at the interface between domains one and two

5C) Formation of a hydrogen bond between the Arginine interaction with the surrounding residues by the with Asn111 and $\pi$ interactions with Tyr320

Fig 6. Superimposition of chosen docked pose of Amprenavir onto the predicted hot-spot map.

\section{Discussion}

The biosynthetic pathways identified as common between $M$. tuberculosis, M. leprae and $M$. abscessus include those for amino acids, carbohydrates, cell structures, aromatic compounds, cofactors, vitamin acids, fatty acids, metabolic regulators, secondary metabolites, as well as nucleoside and nucleotide biosynthetic pathways. These are all necessary for bacterial survival, growth, and division. We did not find the amine and polyamine biosynthetic pathways, which include the ectoine and glutathionylspermidine pathways in $M$. leprae, which were identified in M. abscessus; neither did we find the spermidine pathway in M. leprae, which is present in M. tuberculosis. The biosynthesis of ectoine enables $M$ abscessus, an opportunistic bacterium, to survive in extreme osmotic conditions such as in soil and salt water environments. Also, the glutathionylspermidine biosynthetic pathway in $M$. abscessus and the spermidine biosynthetic pathway present in $M$. tuberculosis might be contributing to the maintenance of redox balance in $M$. abscessus and M. tuberculosis. As expected of an opportunistic organism such as $M$. abscessus, we identified the plant hormone biosynthetic pathway, indole-3-acetate biosynthesis, which is absent in the other two species, $M$. tuberculosis and $M$. leprae, which are obligate human pathogens. 
Our studies on pathway analysis further demonstrate that there are more polysaccharide biosynthetic pathways in $M$. tuberculosis and $M$. abscessus than in $M$. leprae. This complements previous studies by (51), where they showed that other polysaccharides, in addition to cellulose, are key components in the biofilms of M. tuberculosis. Biofilm production, predominant in M. tuberculosis, M. abscessus and Pseudomonas aeruginosa, enables the bacteria to be more tolerant of antibiotics and persist in chronic disease. Species-specific reactions for $M$. tuberculosis that we identified include reactions in the cellulose biosynthetic pathway, although these reactions were missing in the most recent metabolic reconstruction of M. tuberculosis (30).

Common degradation pathways identified in the three species include include those for the turnover of alchohols, amino acids, carbohydrates, fatty acids, as well as those for nucleosides and nucleotides. Of particular note is the degradation of chlorinated compounds, such as dichlorobenzene, which have been observed in $M$. abscessus and $M$. leprae but not in $M$. tuberculosis. Previous studies $(46,47)$ have shown that this pathway is particularly exploited by Pseudomonas spp., which is able to use chlorinated compounds as sole carbon sources for nutrition, and this accounts for the prevalence of Pseudomonas in recreational waters. Therefore, we postulate that an opportunistic bacterium such as $M$. abscessus might be degrading dichlorobenzene as a carbon source whilst it is living in extreme conditions in the soil. We also hypothesize that the presence of a degradation pathway of dichlorobenzene in $M$. leprae suggests that the bacterium is able to survive in non-host environments by exploiting other carbon sources. This is consistent with previous studies $(54,55)$, which showed that the presence of viable $M$. leprae in soil samples is suggestive of environmental reservoirs of the bacteria. The authors, therefore, provided an explanation for the occurrence of leprosy from individuals living in areas where human leprosy cases were not previously reported; this suggests that $M$. leprae might not really be an obligate parasite.

One of the characteristic features of $M$. abscessus is the large number of $A B C$ and $M m p L$ transporters proteins, $\sim 200,(56)$ that are dispersed in the genome (57) compared to that of 31 drug transporters in $M$. tuberculosis and 12 in M. leprae (58). The transporters are a very important set of proteins, involved in lipid, carbohydrate, nucleotide and amino-acid metabolism and enable $M$. abscessus to survive, replicate and maintain immunity within its host. In M. leprae, characterisation of the metabolic pathways is essential to provide insight into the vast array of unknown functions in the reduced genome. Including a comprehensive assembly of the transporter reactions in reconstructing the metabolic network of $M$. abscessus and updating the networks of $M$. tuberculosis and $M$. leprae will improve the in-silico 
predictions of essentiality and provide insight to the effect of drug uptake and resistance observed in M. tuberculosis, M. abscessus and M. leprae.

The above comparative studies using the metabolic pathway databases of $M$. tuberculosis, $M$. abscessus and $M$. leprae have allowed us to identify potential drug targets common to the three species using the chokepoint analysis and to demonstrate the effects of perturbations on the metabolic pathways. The identification of chokepoint reactions within the metabolic network can indicate the essentiality of genes required for the survival of the pathogen. Bringing chokepoint analysis of the reactions together with ligand-binding ability of the enzymes catalysing them is central to the selection of targets for drug discovery. We demonstrate the complementarity of these approaches using the arginine biosynthetic pathway as an example.

The following targets: ArgB, ArgC, ArgD and ArgH in the arginine biosynthetic pathway were among the prioritised drug targets identified through the chokepoint analysis in both $M$. leprae and M. tuberculosis. The four proteins occur in the full list of drug targets identified from the chokepoint analysis of $M$. abscessus. Furthermore, our FBA analysis shows that $\operatorname{ArgB}, \operatorname{Arg} C$, ArgD, ArgG, ArgH and ArgJ are all essential to the bacterium, ArgA amino-acid acetyltransferase, which catalyzes the production of $\mathrm{N}$-acetyl-L-glutamate from L-glutamate is not essential to $M$. tuberculosis and absent in both $M$. leprae and $M$. abscessus. In $M$. tuberculosis, ArgJ can also play a dual function as it possesses the same acetyltransferase as ArgA and can also catalyse the production of L-ornithine from $\mathrm{N}$-acetyl L-glutamate. Having considered all the proteins in the arginine biosynthetic pathway, we selected acetylornithine aminotransferase (ArgD) and argininosuccinate lyase (ArgH), currently with no experimental structures in the Protein Data Bank for further druggability and ligand-binding ability analysis including (i) sub-cellular localization (ii) check using similarity search in DrugBank (iii) comparative 3D-structure analysis of argD in mycobacterial species and humans and (v) pocket analysis (using Fragment Hotspot Maps).

Our analyses indicate that $M$. tuberculosis $\operatorname{ArgD}$ is cytoplasmic and also has significant homologues in DrugBank (with E-value < 0.00001). The druggable potential of ArgD and its applicability in re-purposing drugs was recently demonstrated on a previous study (46), for the identification of polypharmacological targets in M. tuberculosis, using a structural proteomic analysis of binding sites. The authors have comprehensively characterized the pocketome of M. tuberculosis, based on a structural proteomics strategy at genome-scale (46). This study indicates that ArgD in M. tuberculosis (Rv_1655) might be a target for drug-repurposing of compounds such as Amprenavir and Colchicine. Our analyses of pockets and hotspots show 
the Fragment Hotspot mapped around the phosphate moiety of the pyridoxal 5'-phosphate of the ArgD protein on $M$. tuberculosis and at the sub-unit interfaces on domain 2 of the $M$. tuberculosis ArgH protein.

A summary of interaction analysis carried out by PLP is shown in S6 Table and Supp Figure $7 a$ depicts these interactions. Our results suggest that Amprenavir likely interacts with ArgD, whilst a strong interaction with Colchicine is less likely to take place (S6 Table). On the other hand, since Colchicine is a larger and less flexible molecule, docking assays that take receptor flexibility into account could prove to be more reliable. $\mathrm{ArgH}$, on the other hand, demonstrates a promiscuity of interactions and fewer binding sites.

In a previous study, Tiwari and colleagues (59), have described ArgB and ArgJ as potential drug targets. On this study, we have evaluated all the enzymes on the arginine biosynthetic pathway, and provided:

a) The results of the chokepoint analyses (S2 Table) demonstrating that $\mathrm{ArgB}, \mathrm{ArgC}$, ArgD, ArgF, ArgH and ArgJ are potential drug targets in M. tuberculosis, M. leprae and $M$. abscessus.

b) We provide a list of pathways common to the three species, M. tuberculosis, leprae and abscessus, and specie-specific pathways for each mycobacterium (S1 Table).

c) The ligand-binding ability assessment of ArgD and ArgH (Fig 4 and 5) and comparative results with the arginine biosynthetic pathway enzymes in $M$. leprae and M. abscessus.

d) Comparative 3D- models of ArgD and ArgH and descriptions of their fragment hotspot maps.

e) Superimposition of chosen docked pose of Amprenavir onto the predicted hot-spot map of ArgD.

f) Python-based chokepoint algorithm for the drug target identification of a metabolic network file in SBML format and available for download (S1 Text)

Updated metabolic reconstructions of $M$. leprae and $M$. abscessus (Bannerman et al. manuscripts in preparation) will support future analysis for drug repurposing for the benefit of patients affected by infections of $M$. tuberculosis, $M$. leprae and $M$. abscessus. 


\section{Methodology}

\section{Pathway / Chokepoint analysis / Gene Essentiality}

We performed a comparative analysis of pathways in the networks of $M$. tuberculosis, $M$. abscessus and $M$. leprae by using the most recent reconstruction of Mycobacterium tuberculosis metabolic network (30) and the automated build of the pathway genome database for $M$. abscessus and $M$. leprae using the Pathway Tools software (60). To determine potential drug targets, we implemented a pipeline (Fig 1), which includes an in-house chokepoint algorithm in Python, the input of which is a metabolic network expressed in the Systems Biology Markup Language (SBML) format, and the output is a spreadsheet with the reactions that are either the only producers or the only consumers of the metabolites. Metabolites are stored in different sheets according to the number of reactions that produce and consume them, e.g. all the metabolites that are produced by 2 reactions are contained in the same sheet. The sets of reactions that are the only producers, or the only consumers, of a given metabolite are also reported by the algorithm in the same spreadsheet. It should be noted that these sets of reactions can be seen as a generalization of the classical chokepoint algorithm, i.e. if the size of the set of reactions is one, then it corresponds to a chokepoint reaction, if the size is two, then the metabolite is either produced or consumed by at most two reactions, etc. The algorithm also offers the possibility of identifying the 'dead-end' metabolites, i.e. those for which there are no metabolic or transport reactions within the metabolic network, and which are not contained within the final biomass composition equations. Such metabolites indicate that the metabolic model is either incomplete or contains spurious pathways.

This algorithm was run on the M. tuberculosis iEKV1011 metabolic network model and compared with the BioCyc chokepoint implementation (29) on the models of M. tuberculosis, $M$. abscessus and $M$. leprae. We performed searches from literature, the database of essential genes (DEG) (33) and the Online Gene Essentiality (OGEE) (34) database to determine the in vivo and in vitro essentiality of the potential targets. We also performed Flux Balance Analysis (FBA) and Flux Variability Analysis (FVA) on the M. tuberculosis iEK1011 model (30) using the Python toolbox COBRApy (61).

\section{Structure modelling of selected drug targets}

Comparative modelling of selected drug targets, $\mathrm{ArgD}$ and $\mathrm{ArgH}$, chokepoint enzymes of the arginine biosynthetic pathway, which currently lack experimental structures in the PDB, was performed using an in-house modelling pipeline, Vivace (Ochoa M. et al., Skwark M. and Blundell TL. manuscript in preparation). The pipeline was built using the Ruffus (Python) (Goodstadt, 2010) architecture that performs multiple tasks to generate models with 
substantial quality and reliability. In the modelling process, templates were selected from TOCCATA (Ochoa Montaño B, Blundell TL, unpublished) a database of consensus profiles built from CATH v4.2.0 and SCOPe v2.07 $(62,63)$ based classification of protein structures (PDB files). PDBs within each profile are clustered based on sequence similarity using CDHIT $(64,65))$ and structures are aligned using BATON, which was developed as a streamlined version of the in-house program COMPARER (66). Later FUGUE (63) is employed to generate target-template alignments from different profiles and profiles with the highest Z-scores are used in the downstream process. After further optimization of the clusters by discarding templates with more than $20 \%$ difference in sequence identity to the maximum hit, the remaining templates are classified into states based on ligand binding. Different states were generated for ligand-binding and one state for apomeric structure. Models are built for each of these states using MODELLER (36) version 9.19 (37).

Druggability checks of the selected targets, such as subcellular localization, were predicted using PSORTb a database of protein subcellular localization for bacteria and archaea (68), subCELlular LOcalization predictor, CELLO (69), TB-Pred (70). The identified target sequences were also searched against databases such as Drug Bank (target sequence collection for approved drugs) (71) and Therapeutic Target Database (TTB) (72), using default parameter settings of BLAST. The identification of significant hits in such databases is an indicator of the potential evidence of druggability of the target. AutoDock Vina was then used to investigate the potential interactions of suggested drug candidates with the proteins using a molecular docking approach (73).

\section{Acknowledgements}

We thank the following funding agencies for providing support to the Blundell and Oliver groups. JJ: the Industrial Biotechnology Catalyst (Innovate UK, BBSRC, EPSRC) to support the translation, development and commercialisation of innovative Industrial Biotechnology processes; SCV: American Leprosy Mission; MJS: The Botnar Foundation (Project 6063); PT: The Cystic Fibrosis Trust (Strategic Centre Award-201); SM: the UK Medical Research Council (MRC-DBT Centre Partnership); AM: The Cambridge Commonwealth Trust (10380426) and the Pakistan HEC Cambridge Scholarship. The authors also thank Dr Bill Jacobs for useful discussions. 


\section{References}

1. Singh P, Benjak A, Schuenemann VJ, Herbig A, Avanzi C, Busso P, et al. Insight into the evolution and origin of leprosy bacilli from the genome sequence of Mycobacterium lepromatosis. Proceedings of the National Academy of Sciences of the United States of America. 2015 Apr 7;112(14):4459-64.

2. Schuenemann VJ, Singh P, Mendum TA, Krause-Kyora B, Jäger G, Bos KI, et al. Genome-Wide Comparison of Medieval and Modern $<$ em $>$ Mycobacterium leprae $</$ em>. Science. 2013 Jul 12;341(6142):179.

3. Warner D. Mycobacterium tuberculosis metabolism. Cold Spring Harb Perspect Med. 2015;5(4):a021121.

4. Bryant J, Grogono D, Rodriguez-Rincon D, Everall I, Brown K, Moreno P, et al. Population-level genomics identifies the emergence and global spread of a human transmissible multidrug-resistant nontuberculous mycobacterium. Science.

2016;354:751.

5. Gupta RS, Lo B, Son J. Phylogenomics and Comparative Genomic Studies Robustly Support Division of the Genus Mycobacterium into an Emended Genus Mycobacterium and Four Novel Genera. Frontiers in Microbiology. 2018;9:67.

6. Medjahed H, Gaillard J-L, Reyrat J-M. Mycobacterium abscessus: a new player in the mycobacterial field. Trends in Microbiology. 2010 Mar 1;18(3):117-23.

7. Alberts B, Johnson A, Lewis J, et al. Molecular Biology of the Cell. New York: Garland Science. 2002;4th edition.

8. de Bolster M. "Glossary of Terms Used in Bioinorganic Chemistry: Catabolism". International Union of Pure and Applied Chemistry. Archived from the original on 2017-01-21. 1997;

9. Rhee K. Minding the gaps: metabolomics mends functional genomics. EMBO Rep. 2013;14(11):949-50.

10. Muñoz-Elías E, McKinney J. Carbon metabolism of intracellular bacteria. Cell Microbiol. 2006;8(1):10-22.

11. Beste D, McFadden J. System-level strategies for studying the metabolism of Mycobacterium tuberculosis. Mol Biosyst. 2010;6(12):2363-72.

12. Wheeler P. The microbial physiologist's guide to the leprosy genome. Lepr Rev. 2001;72(4):399-407.

13. Cole ST, Brosch R, Parkhill J, Garnier T, Churcher C, Harris D, et al. Deciphering the biology of Mycobacterium tuberculosis from the complete genome sequence. Nature. 1998 Jun 11;393:537.

14. Bringel, F, Frey, L, Boivin, S, Hubert JC. Arginine Biosynthesis and Regulation in Lactobacillus plantarum: the carA Gene and the argCJBDF Cluster Are Divergently Transcribed. JOURNAL OF BACTERIOLOGY. 1997;179(8):2697-2706. 
15. Cunin, R, Glansdorff N, Piérard, A, Stalon, S. Biosynthesis and metabolism of arginine in bacteria. Microbiol Rev. 1986;50(3):314-352.

16. Gordhan B G, Smith D, Alderton H, McAdam R A, Bancroft G J, Mizrahi V. Construction and Phenotypic Characterization of an Auxotrophic Mutant of Mycobacterium tuberculosis Defective in 1-Arginine Biosynthesis. Infect Immun. 2002;70(6):3080-3084.

17. Raman K, Yeturu K, Chandra N. targetTB: A target identification pipeline for Mycobacterium tuberculosis through an interactome, reactome and genome-scale structural analysis. BMC Syst Biol. 2008;2:109.

18. Cherney LT, Cherney MM, Garen CR, Niu C, Moradian F, James MNG. Crystal Structure of N-acetyl- $\gamma$-glutamyl-phosphate Reductase from Mycobacterium tuberculosis in Complex with NADP+. Journal of Molecular Biology. 2007 Apr 13;367(5):1357-69.

19. Ruepp A, Soppa J. Fermentative arginine degradation in Halobacterium salinarium (formerly Halobacterium halobium): genes, gene products, and transcripts of the arcRACB gene cluster. Journal of bacteriology. 1996 Aug;178(16):4942-7.

20. Arena ME, Saguir FM, Manca de Nadra MC. Arginine, citrulline and ornithine metabolism by lactic acid bacteria from wine. International Journal of Food Microbiology. 1999 Nov 15;52(3):155-61.

21. Tabor CW, Tabor H. Polyamines in microorganisms. Microbiological reviews. 1985 Mar;49(1):81-99.

22. Burley S, Berman H, Kleywegt G, Markley J, Nakamura H, Velankar S. Protein Data Bank (PDB): The Single Global Macromolecular Structure Archive. Methods Mol Biol. 2017;1607:627-41.

23. Errey J, Blanchard J. Functional characterization of a novel ArgA from Mycobacterium tuberculosis. J Bacteriol. 2005;187(9):3039-44.

24. Sankaranarayanan R, Garen C, Cherney M, Yuan M, Lee C, James M. Preliminary X-ray crystallographic analysis of ornithine acetyltransferase (Rv1653) from Mycobacterium tuberculosis. Acta Crystallogr Sect F Struct Biol Cryst Commun. 2009;65((Pt 2)):173-6.

25. Sankaranarayanan R, Garen C, Cherney M, Yuan M, Lee C, James M. The molecular structure of ornithine acetyltransferase from Mycobacterium tuberculosis bound to ornithine, a competitive inhibitor. JMolBiol. 2010;397:979-90.

26. Thiele I, Palsson BØ. A protocol for generating a high-quality genome-scale metabolic reconstruction. Nature protocols. 2010;5(1):93-121.

27. Chavali AK, D'Auria KM, Hewlett EL, Pearson RD, Papin JA. A metabolic network approach for the identification and prioritization of antimicrobial drug targets. Trends in microbiology. 2012 Mar;20(3):113-23.

28. Lee D-S, Burd H, Liu J, Almaas E, Wiest O, Barabási A-L, et al. Comparative genomescale metabolic reconstruction and flux balance analysis of multiple Staphylococcus 
aureus genomes identify novel antimicrobial drug targets. Journal of bacteriology. 2009 Jun;191(12):4015-24.

29. Yeh I, Hanekamp T, Tsoka S, Karp P, Altman R. Computational analysis of Plasmodium falciparum metabolism: organizing genomic information to facilitate drug discovery. Genome Res. 2004;14:917-924.

30. Kavvas E, Seif Y, Yurkovich J. Norsigian C, Poudel S, Greenwald WW, et al. Updated and standardized genome-scale reconstruction of Mycobacterium tuberculosis H37Rv, iEK1011, simulates flux states indicative of physiological conditions. BMC Syst Biol. 2018;12:25.

31. Ripoll F, Pasek S, Schenowitz C, Dossat C, Barbe V, Rottman M, et al. Non mycobacterial virulence genes in the genome of the emerging pathogen Mycobacterium abscessus. PloS one. 2009 Jun 19;4(6):e5660-e5660.

32. Singh P, Cole S. Mycobacterium leprae: genes, pseudogenes and genetic diversity. Future Microbiol. 2011;6(1):57-71.

33. Luo H, Lin Y, Gao F, Zhang C-T, Zhang R. DEG 10, an update of the Database of Essential Genes that includes both protein-coding genes and non-coding genomic elements. Nucleic Acids Research. 2014;42:D574-80.

34. Chen W, Minguez P, Lercher M, Bork P. OGEE: an online gene essentiality database. Nucleic Acids Res. 2012;40(Database issue):D901-6.

35. Orth JD, Thiele I, Palsson BØ. What is flux balance analysis? Nat Biotechnol. 2010;28(3):245-248.

36. Sali A, Blundell T. Comparative protein modelling by satisfaction of spatial restraints. J Mol Biol. 1993;234:779-815.

37. Webb B, Sali A. Comparative Protein Structure Modeling Using Modeller. Current Protocols in Bioinformatics. 2016;54(1):5.6.1-5.6.37.

38. Davis I, Murray L, Richardson J, Richardson D. MOLPROBITY: structure validation and all-atom contact analysis for nucleic acids and their complexes. Nucleic Acids Res. 2004;32:W615-619.

39. Chen et al. MolProbity: all-atom structure validation for macromolecular crystallography. Acta Crystallographica. 2010;D66:12-21.

40. Jaidhan B, Rao S, Apparao A. Energy Minimization and Conformation Analysis of Molecules using Steepest Descent Method. International Journal of Computer Science and Information Technologies. 2014;5(3):3525-8.

41. Robbins H, Monro S. A Stochastic Approximation Method. The Annals of Mathematical Statistics. 1951;22(3):400.

42. Xu D, Zhang Y. Improving the Physical Realism and Structural Accuracy of Protein Models by a Two-step Atomic-level Energy Minimization. Biophysical Journal. 2011;101:2525-34. 
43. Radoux C, Olsson TS., Pitt W, Groom C, Blundell T. Identifying the Interactions that Determine Fragment Binding at Protein Hotspots. J Med Chem. 2016;59(9):4314-25.

44. Mizuguchi K, Deane C, Blundell T, Johnson M, Overington J. JOY: protein sequencestructure representation and analysis. Bioinforma Oxf Engl. 1998;14:617-623.

45. Mishra A, Surolia A. Biochemical characterization of argininosuccinate lyase from $M$. tuberculosis: significance of a c-terminal cysteine in catalysis and thermal stability. IUBMB Life. 2017;69:896-907.

46. Anand P, Chandra N. Characterizing the pocketome of Mycobacterium tuberculosis and application in rationalizing polypharmacological target selection. Sci Rep. 2014;4:6356.

47. Swanson R. The Entrance of Informatics into Combinatorial Chemistry". 2004;

48. Frishman D, Argos P. Knowledge-based secondary structure assignment. Proteins: structure, function and genetics. 1995;23:566-79.

49. Pronk S, Páll S, Schulz R, Larsson P, Bjelkmar P, Apostolov R, et al. GROMACS 4.5: a high-throughput and highly parallel open source molecular simulation toolkit. Bioinformatics. 2013;29(7):845-54.

50. Sander T, Freyss J, von Korff M, Rufener C. DataWarrior: an open-source program for chemistry aware data visualization and analysis. J Chem Inf Model. 2015;55(2):460-73.

51. Kumar A. House of cellulose - a new hideout for drug tolerant Mycobacterium tuberculosis. Microb Cell. 2016;3(7):299-301.

52. van der Meer JR, van Neerven AR, de Vries EJ, de Vos WM, Zehnder AJ. Cloning and characterization of plasmid-encoded genes for the degradation of 1,2-dichloro-, 1,4dichloro-, and 1,2,4-trichlorobenzene of Pseudomonas sp. strain P51. Journal of bacteriology. 1991 Jan;173(1):6-15.

53. Potrawfke T, Timmis K, Wittich R. Degradation of 1,2,3,4-tetrachlorobenzene by pseudomonas chlororaphis RW71. Applied and environmental microbiology. 1998 Oct;64(10):3798-806.

54. Tió-Coma M, Wijnands T, Pierneef L, Schilling AK, Alam K, Roy JC, et al. Detection of Mycobacterium leprae DNA in soil; Multiple needles in the haystack? bioRxiv. 2018 Jan $1 ; 367219$.

55. Lavania M, Katoch K, Katoch V, Gupta A, Chauhan D, Sharma R, et al. Detection of viable Mycobacterium leprae in soil samples: Insights into possible sources of transmission of leprosy. Infection, Genetics and Evolution. 2008;8(5):627-31.

56. Torres P, Skwark M, Copoiu L, Bannerman B, Blundell T. Mabellini: An on-line source for Mycobacterium abscessus modelled structural proteome. Manuscript in preparation.

57. Nessar R, Cambau E, Reyrat JM, Alan Murray, Gicquel B. Mycobacterium abscessus: a new antibiotic nightmare. Journal of Antimicrobial Chemotherapy. 67(4):810-818. 
58. Youm J, Saier MJ. Comparative analyses of transport proteins encoded within the genomes of Mycobacterium tuberculosis and Mycobacterium leprae. Biochim Biophys Acta. 2012;1818(3):776-97.

59. Tiwari S, Tonder A, Vilcheze C, Mendes V, Thomas S, Malek A, et al. Argininedeprivation-induced oxidative damage sterilizes Mycobacterium tuberculosis. Proceedings of the National Academy of Sciences. 2018 Aug;pnas. 1808874115.

60. Karp PD, Latendresse M, Paley SM, Krummenacker M, Ong QD, Billington R, et al. Pathway Tools version 19.0 update: software for pathway/genome informatics and systems biology. Briefings in Bioinformatics. 2016 Sep 1;17(5):877-90.

61. Ebrahim A, Lerman JA, Palsson BO, Hyduke DR. COBRApy: COnstraints-Based Reconstruction and Analysis for Python. BMC Systems Biology. 2013;2013:7:74.

62. Orengo C, Michie A, Jones S, Jones D, Swindells M, Thornton J. CATH - a hierarchic classification of protein domain structures. Structure. 1997 Aug 15;5(8):1093-109.

63. Sillitoe I, Dawson N, Lewis TE, Das S, Lees JG, Ashford P, et al. CATH: expanding the horizons of structure-based functional annotations for genome sequences. Nucleic Acids Research. 2018 Nov 6;gky1097-gky1097.

64. Li W, Godzik A. Cd-hit: a fast program for clustering and comparing large sets of protein or nucleotide sequences. Bioinformatics. 2006;22:1658-9.

65. Fu L, Niu B, Zhu Z, Wu S, Li W. CD-HIT: accelerated for clustering the next generation sequencing data. Bioinformatics. 2012;28(23):3150-2.

66. Johnson M, Overington J, Blundell T. Alignment and searching for common protein folds using a Data Bank of structural templates. JMB. 1993;231:735-52.

67. Shi J, Blundell T, Mizuguchi. FUGUE: sequence-structure homology recognition using environment-specific substitution tables and structure-dependent gap penalties. J Mol Biol. 2001;310:243-257.

68. Yu NY, Wagner JR, Laird MR, Melli G, Rey S, Lo R, et al. PSORTb 3.0: improved protein subcellular localization prediction with refined localization subcategories and predictive capabilities for all prokaryotes. Bioinformatics. 2010;26(13):1608-1615.

69. Yu C, Chen Y, Lu C, Hwang J. Prediction of protein subcellular localization. Proteins. 2006;64(3):643-51.

70. Rashid M, Saha S, Raghava G. Support Vector Machine-based method for predicting subcellular localization of mycobacterial proteins using evolutionary information and motifs. BMC Bioinformantics. 2007;8:337.

71. Wishart D. DrugBank 5.0: a major update to the DrugBank database for 2018. Nucleic Acids Res. 2018;46(D1):D1074-D1082. doi: 10.1093/nar/gkx1037. Wishart DS, Feunang YD, Guo AC, Lo EJ, Marcu A, Grant JR, Sajed T, Johnson D, Li C, Sayeeda Z, Assempour N, Iynkkaran I, Liu Y, Maciejewski A, Gale N, Wilson A, Chin L, Cummings R, Le D, Pon A, Knox C, Wilson M. 
72. Li Y. Therapeutic target database update 2018: enriched resource for facilitating benchto-clinic research of targeted therapeutics. Nucleic Acids Res. 2018 ;46(D1):D1121D1127. doi: 10.1093/nar/gkx1076. Li YH, Yu CY, Li XX, Zhang P, Tang J, Yang Q, Fu T, Zhang X, Cui X, Tu G, Zhang Y, Li S, Yang F, Sun Q, Qin C, Zeng X, Chen Z, Chen YZ, Zhu F.

73. Trott O, Olson A. AutoDock Vina: improving the speed and accuracy of docking with a new scoring function, efficient optimization, and multithreading. J Comput Chem. 2010;31(2):455-61.

\section{Supporting Information}

S1 Fig 1A. JOY Alignment of Human ArgD (2BYJ) with Mtb ArgD Model

S1 Fig 1B Superimposition of chain A of Mtb ArgD (Orange) with chain A of Human ArgD (Blue)

S2 Fig 2A. JOY Alignment of Human ArgH (1AOS) with Mtb ArgH Model

S2. Fig 2B. Superimposition of chain A of $M t b \mathrm{ArgH}$ with chain A of Human ArgH (1AOS)

S3. Fig 3A. Three different hotspot maps in Mtb ArgD Model

S3 Fig 3B. One Hotspot map adjacent to the PLP binding site in Human ArgD (2BYJ)

S3 Fig 3c: Hotspot Maps on human ArgD 2BYJ and M tb ArgD

S4. Fig 4A. Three hotspot maps at the subunit interfaces in Mtb ArgH

S4. Fig 4B. Hotspot maps at the subunit interfaces in Human ArgH (1AOS)

S5. Fig 5A. Clustering of docking poses for the docked molecules - PLP

S5. Fig 5B. Clustering of docking poses for the docked molecules - Amprenavir

S5. Fig 5C. Clustering of docking poses for the docked molecules - Colchicine.

S6. Fig 6. Superimposition of chosen docked pose of Piridoxal 5' Phosphate (in green) and the orientation obtained by X-ray crystallography (in cyan) after structural alignment of the experimental and modelled protein structures.

S1 Table. Summary of pathways present in M. tuberculosis, M. abscessus and M. leprae S2 Table. Chokepoint reactions present in M. tuberculosis (A), M. abscessus (B) and $M$. leprae (C)

S3 Table 3. Maximum growth rates (h-1) enzymes of the arginine biosynthetic pathway predicted by FBA

S4 Table 4. Sequence Structure comparisons between human and Mtb ArgD and ArgH S5 Table 5. PSI-BLAST of Mtb ArgH with human proteome

S6 Table 6. Interaction analysis carried out by PLP

S1 File. This is the Chokepoint algorithm 


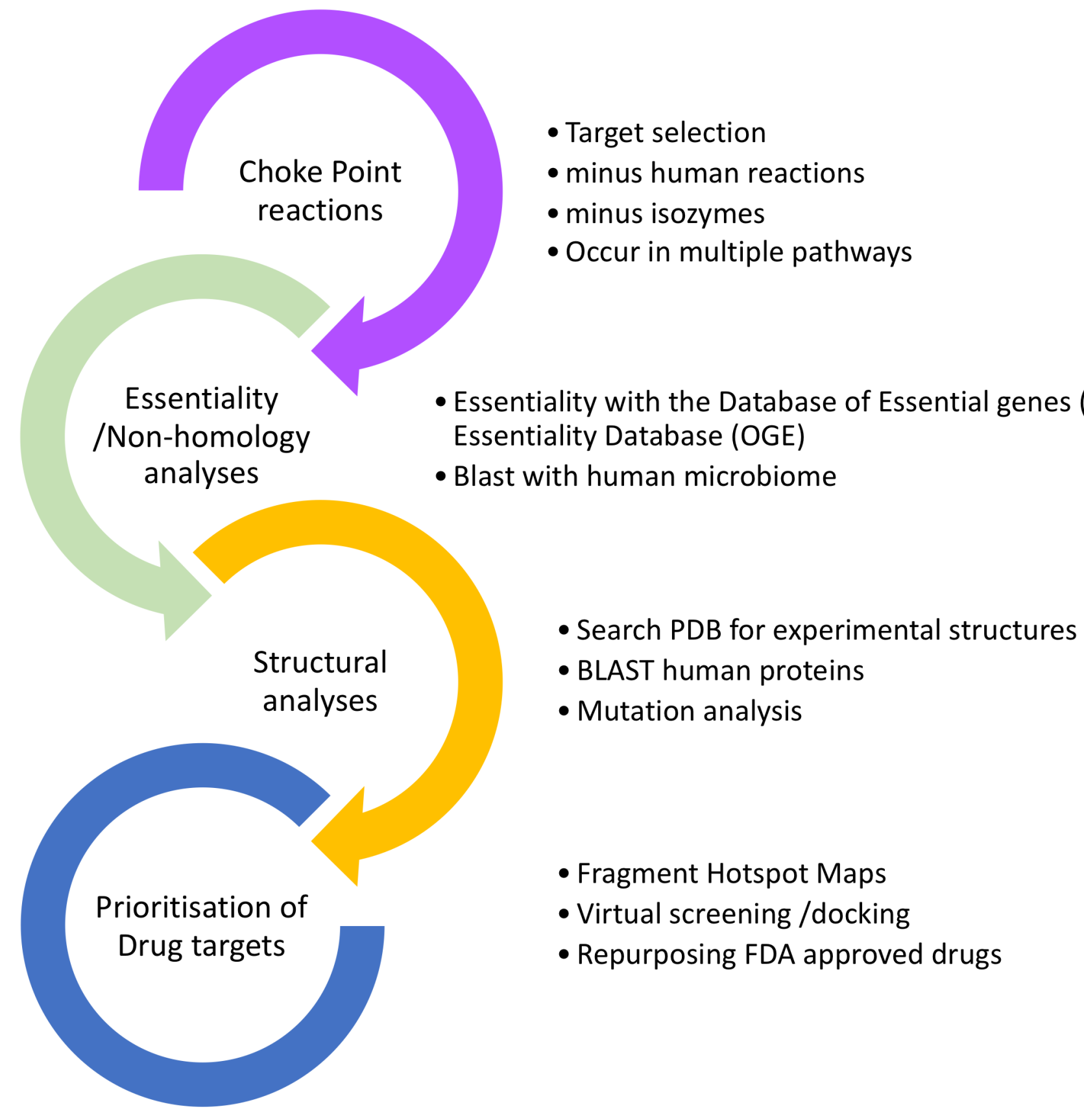

Fig 1: Pipeline for drug target identification in mycobacteria 
Mycobacterium tuberculosis H37Rv Cellular Overview

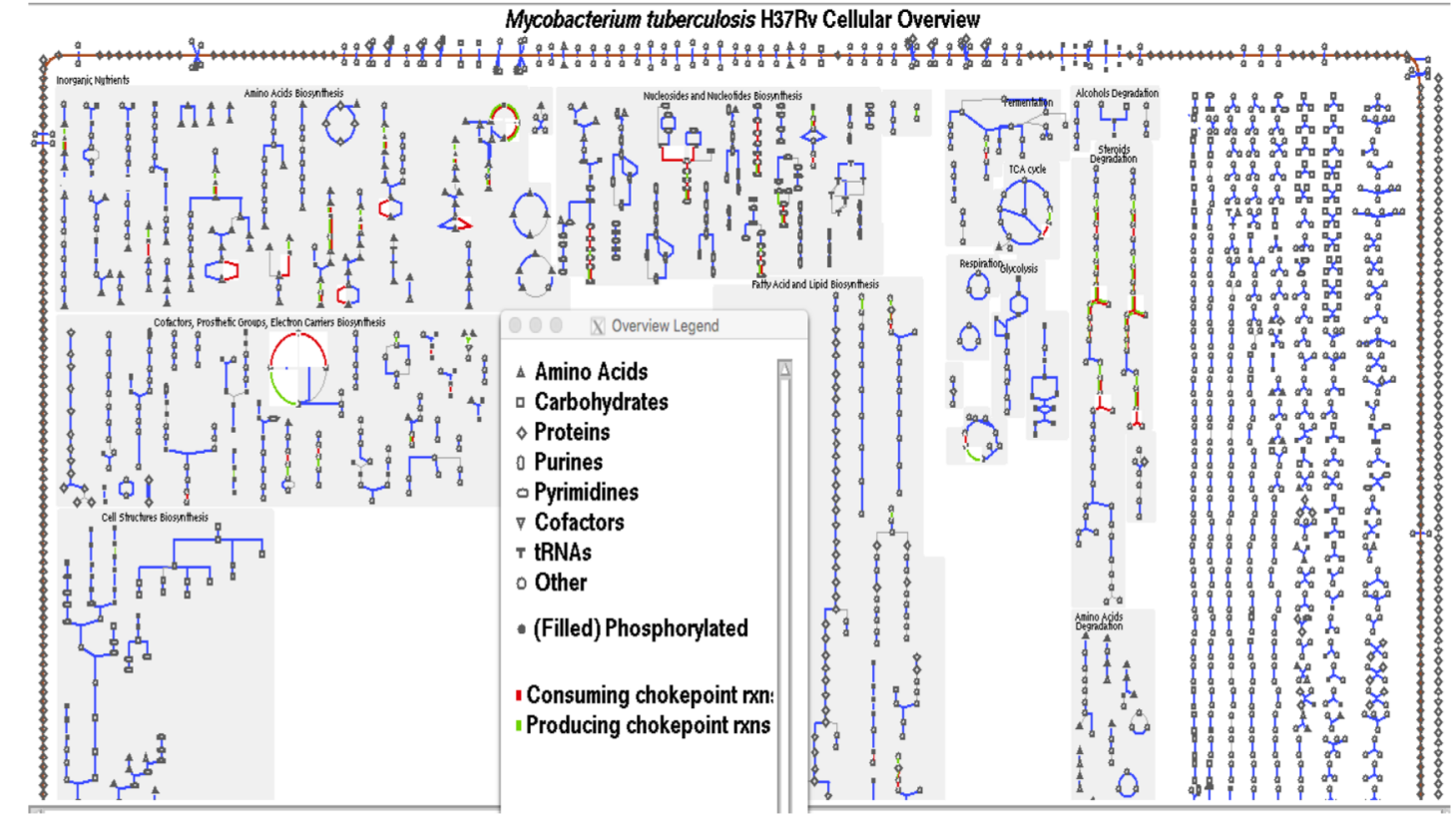

Mycobacterium leprae TN Cellular Overview

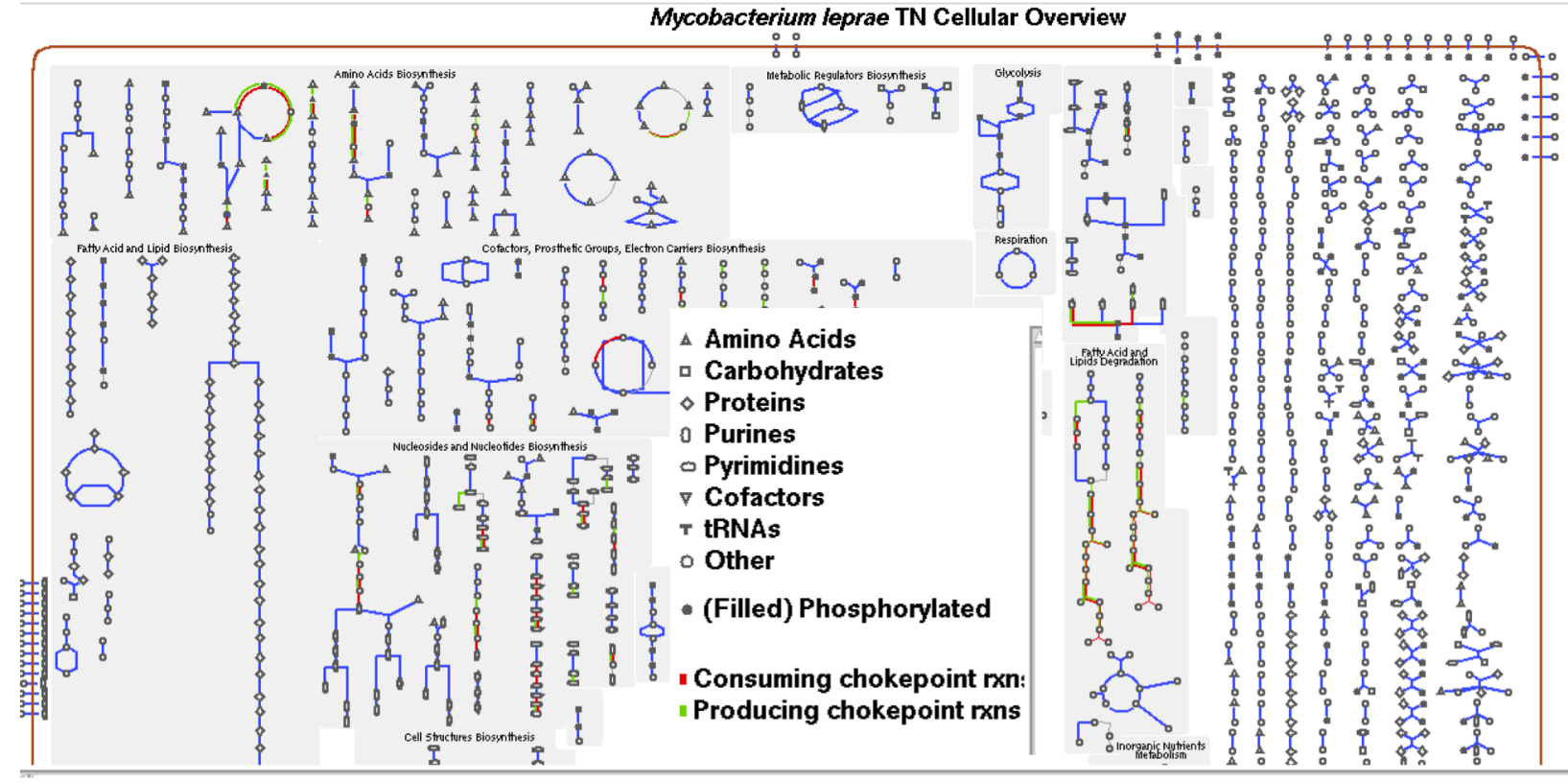

Mycobacterium abscessus Cellular Overview

\begin{tabular}{|c|c|c|}
\hline 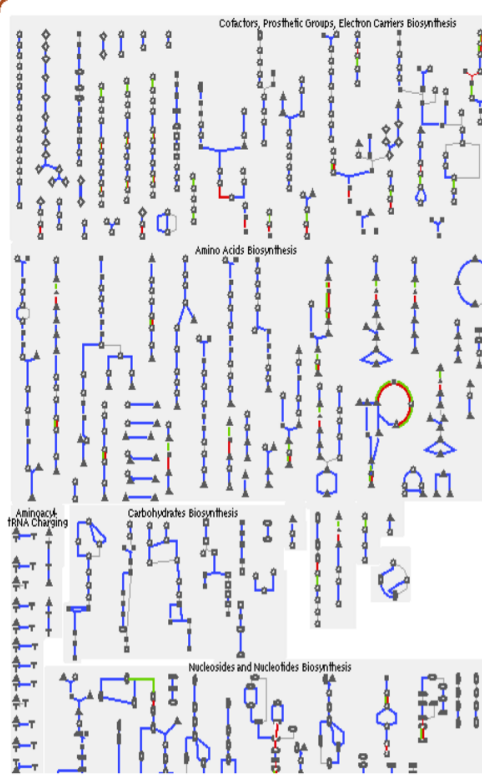 & $\begin{array}{l}\text { A Amino Acids } \\
\text { a Carbohydrates } \\
\text { Proteins } \\
\text { o Purines } \\
\text { a Pyrimidines } \\
\nabla \text { Cofactors } \\
\text { T tRNAs } \\
\text { o Other } \\
\text { - (Filled) Phosphorylated }\end{array}$ & 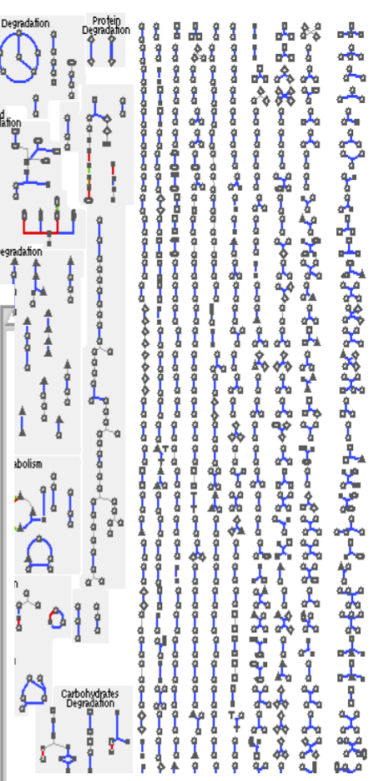 \\
\hline
\end{tabular}

Fig 2: Choke point reactions in mycobacteria 

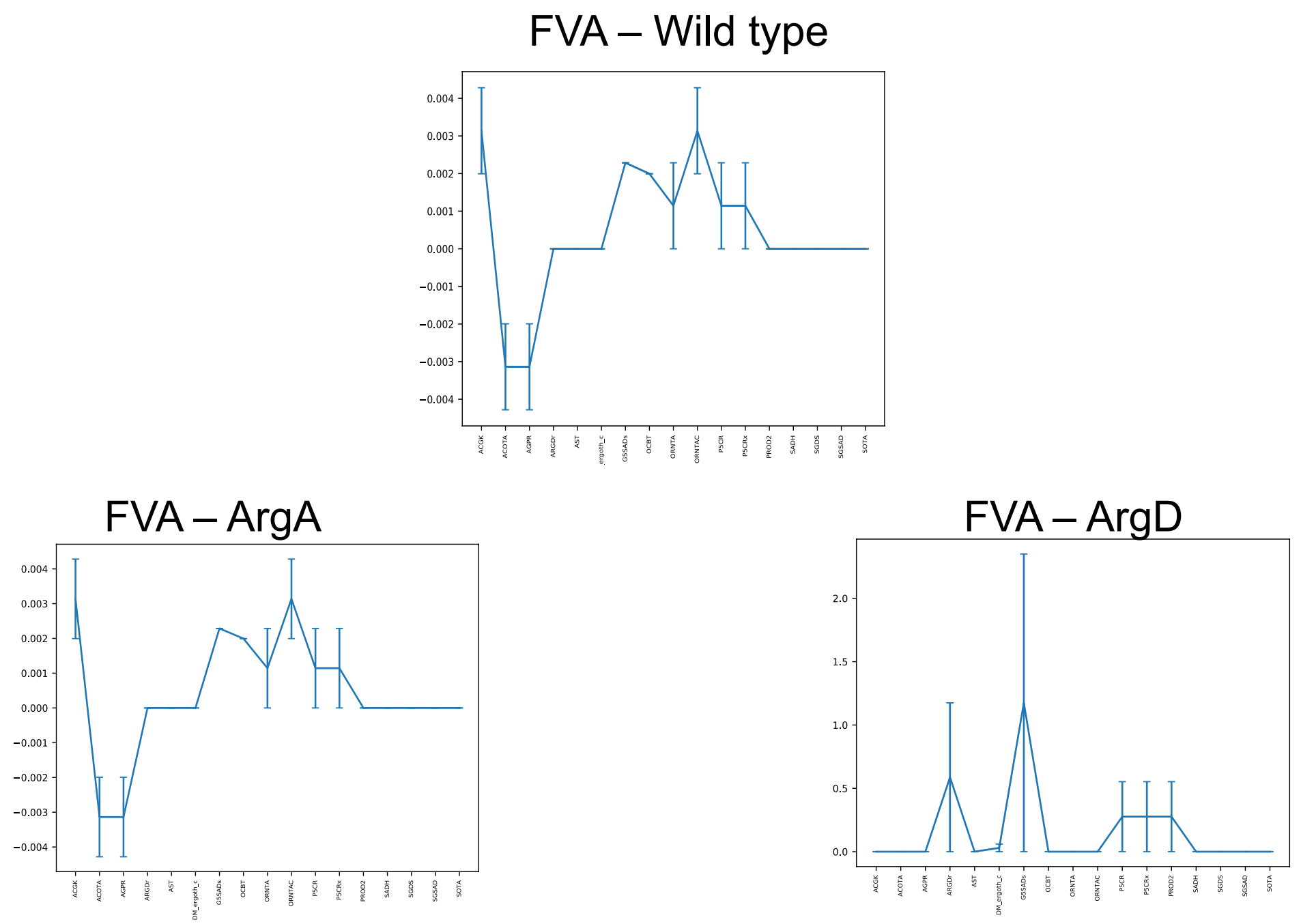

Fig 3: Flux Variability Analysis (FVA) demonstrates the difference in the flux of reactions in the arginine Biosynthetic pathway when enzymes, ArgA and ArgD are absent 

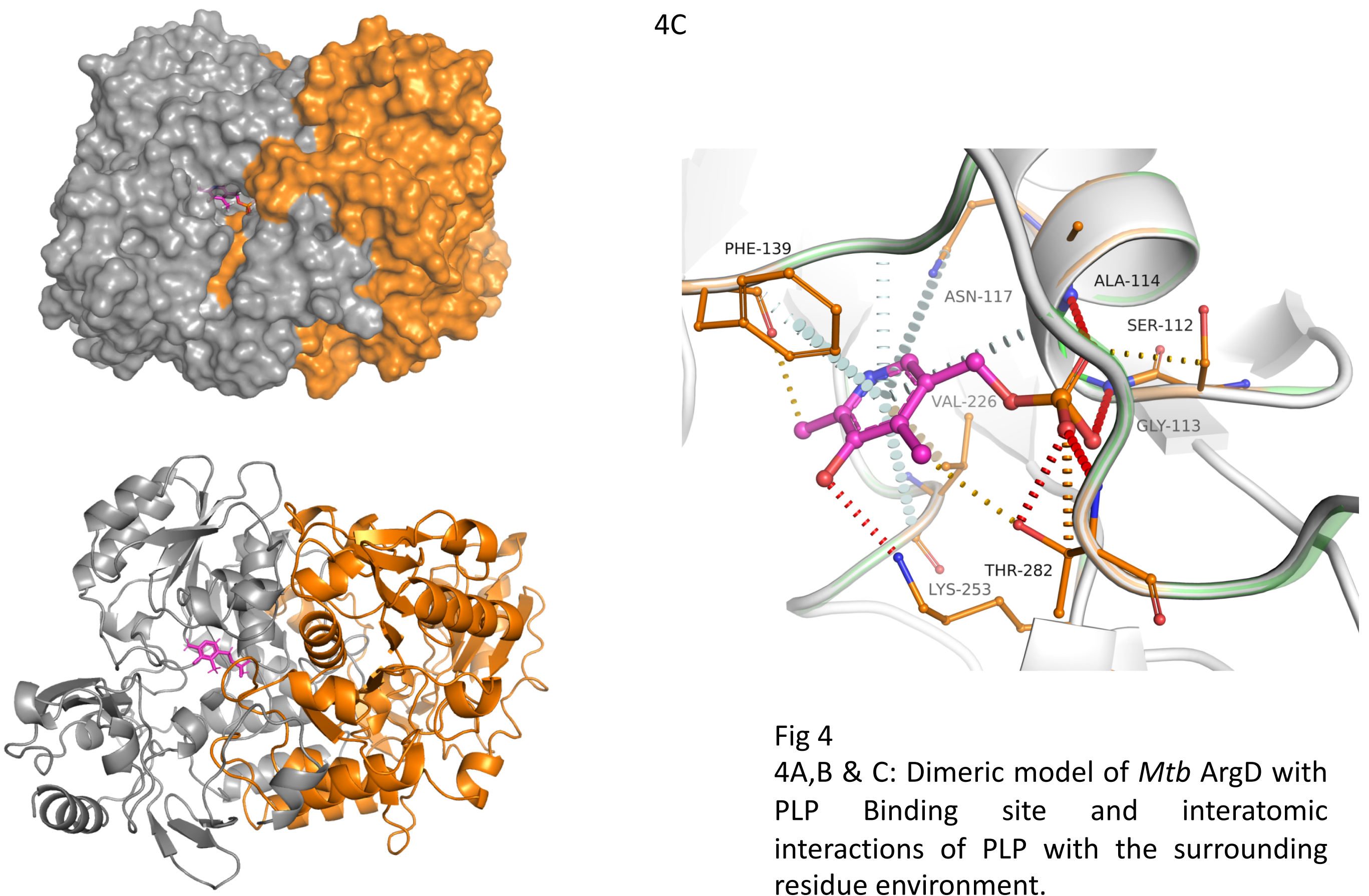

Fig 4

4A,B \& C: Dimeric model of Mtb ArgD with PLP Binding site and interatomic interactions of PLP with the surrounding residue environment. 

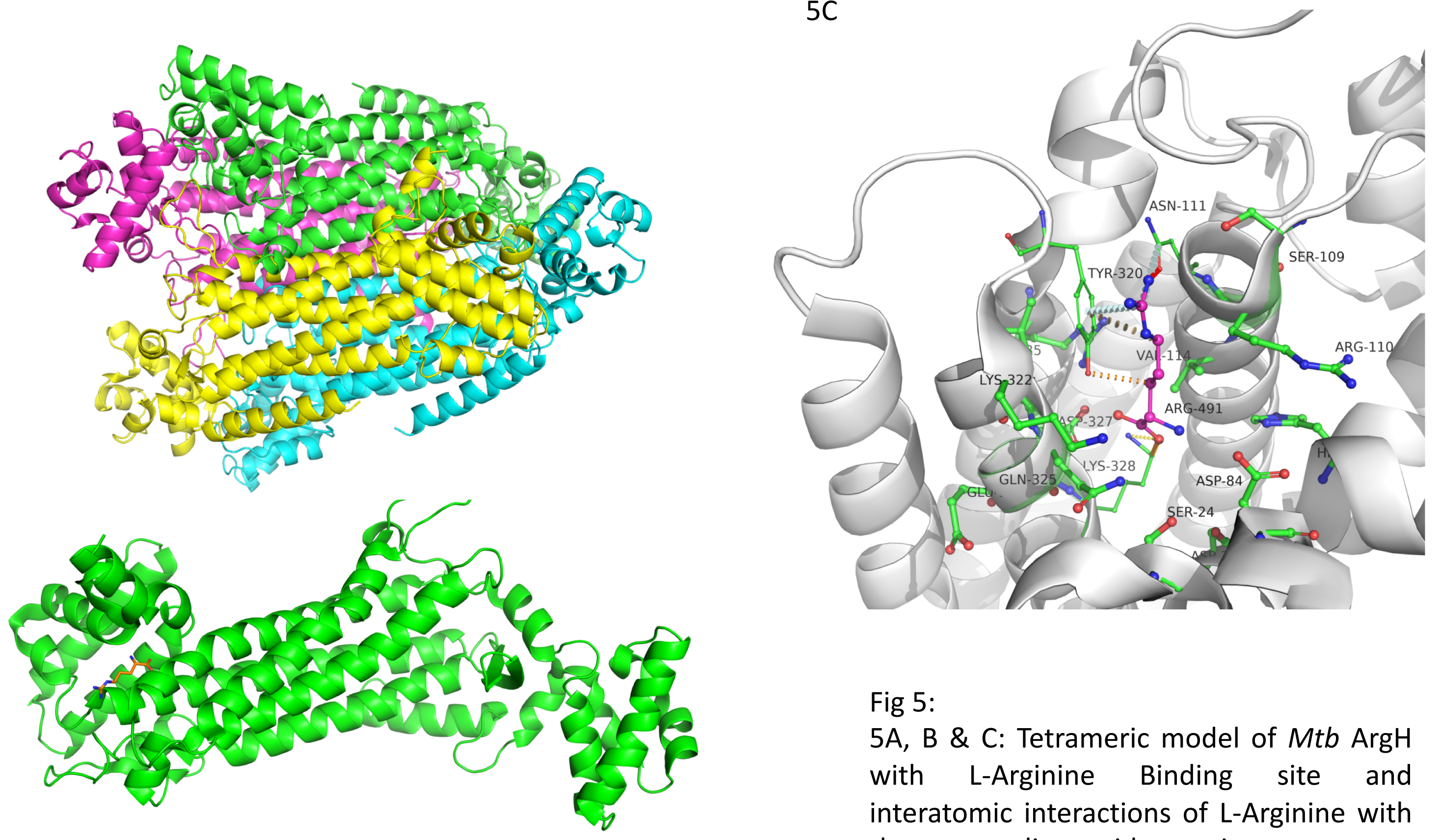

Fig 5:

5A, B \& C: Tetrameric model of Mtb ArgH with L-Arginine Binding site and interatomic interactions of L-Arginine with the surrounding residue environment. 


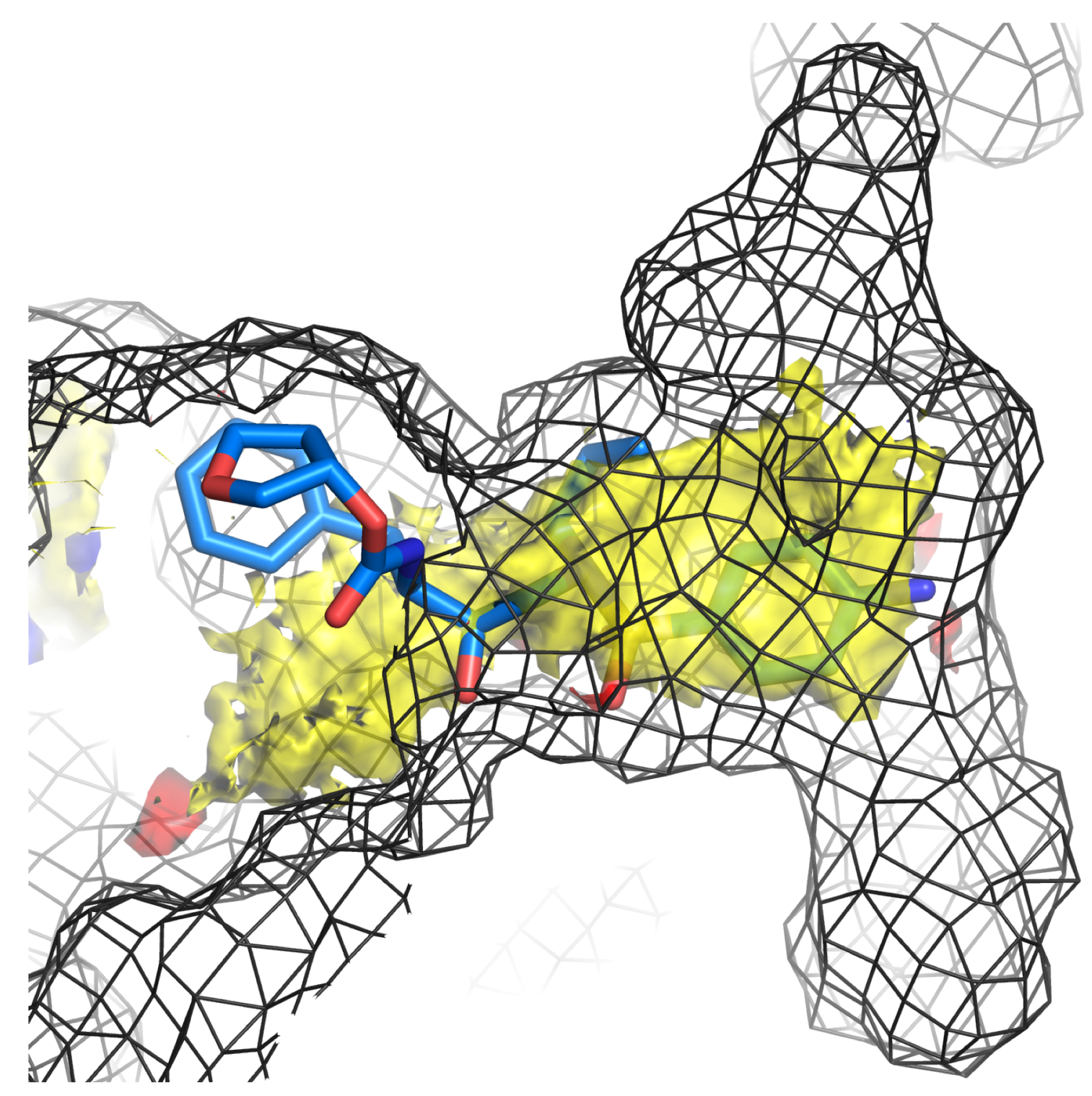

Fig 6 - Superimposition of chosen docked pose of Amprenavir onto the predicted hot-spot map. The protein binding site volume is represented as dark-grey mesh. 


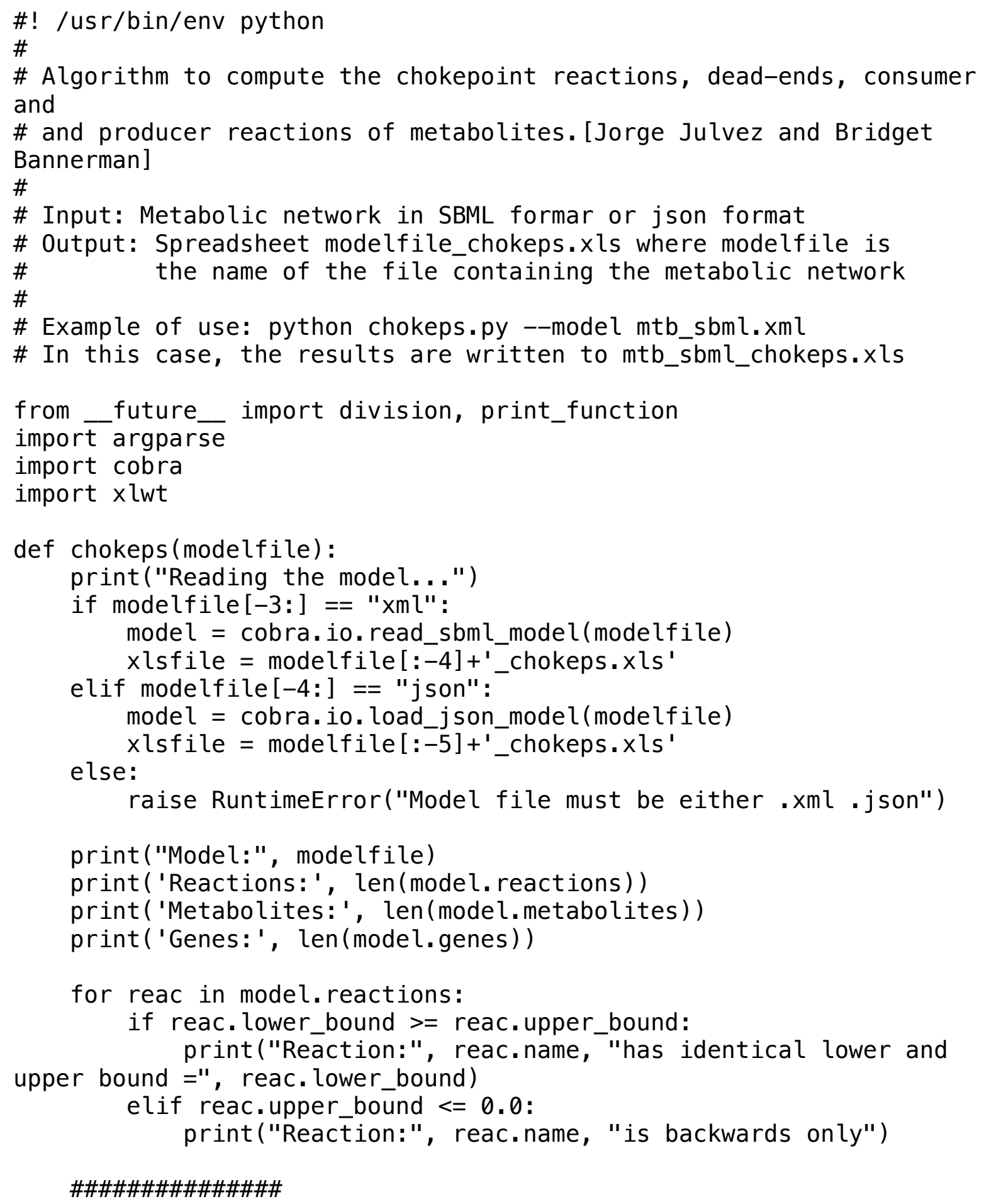

chokedict $=\{-1:[]\} \#-1$ is the key of the dictionary for chokepoints

for meta in model.metabolites:

influxes, outfluxes $=[],[]$

for reac in meta. reactions:

if reac. lower_bound $>=0.0$ : \# forward reaction only

if reac.metabolites [meta] > 0 : \# influx influxes.append (reac.id)

elif reac.metabolites [meta] < 0 : \# outflux outfluxes. append ( reac.id) 


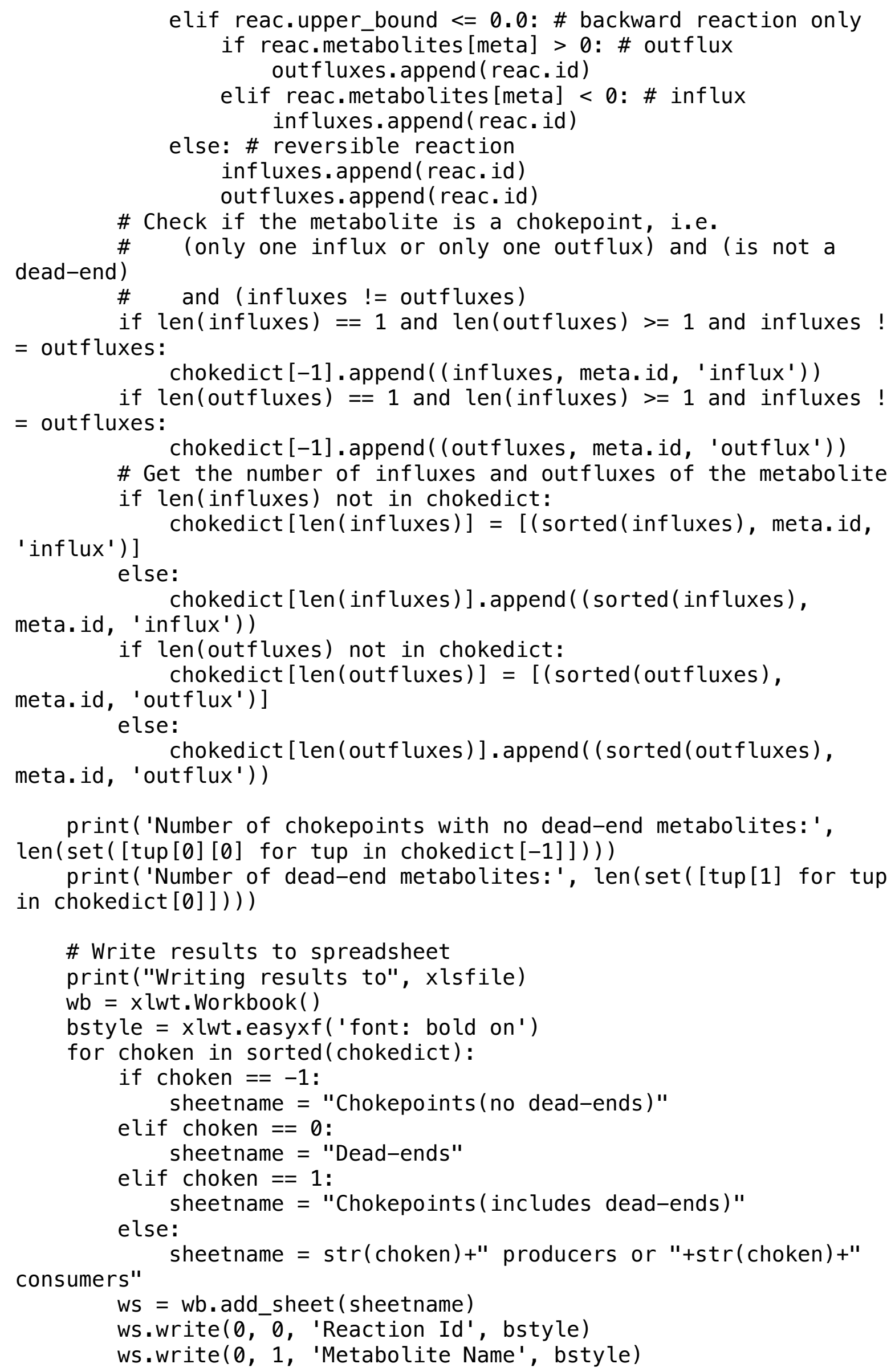




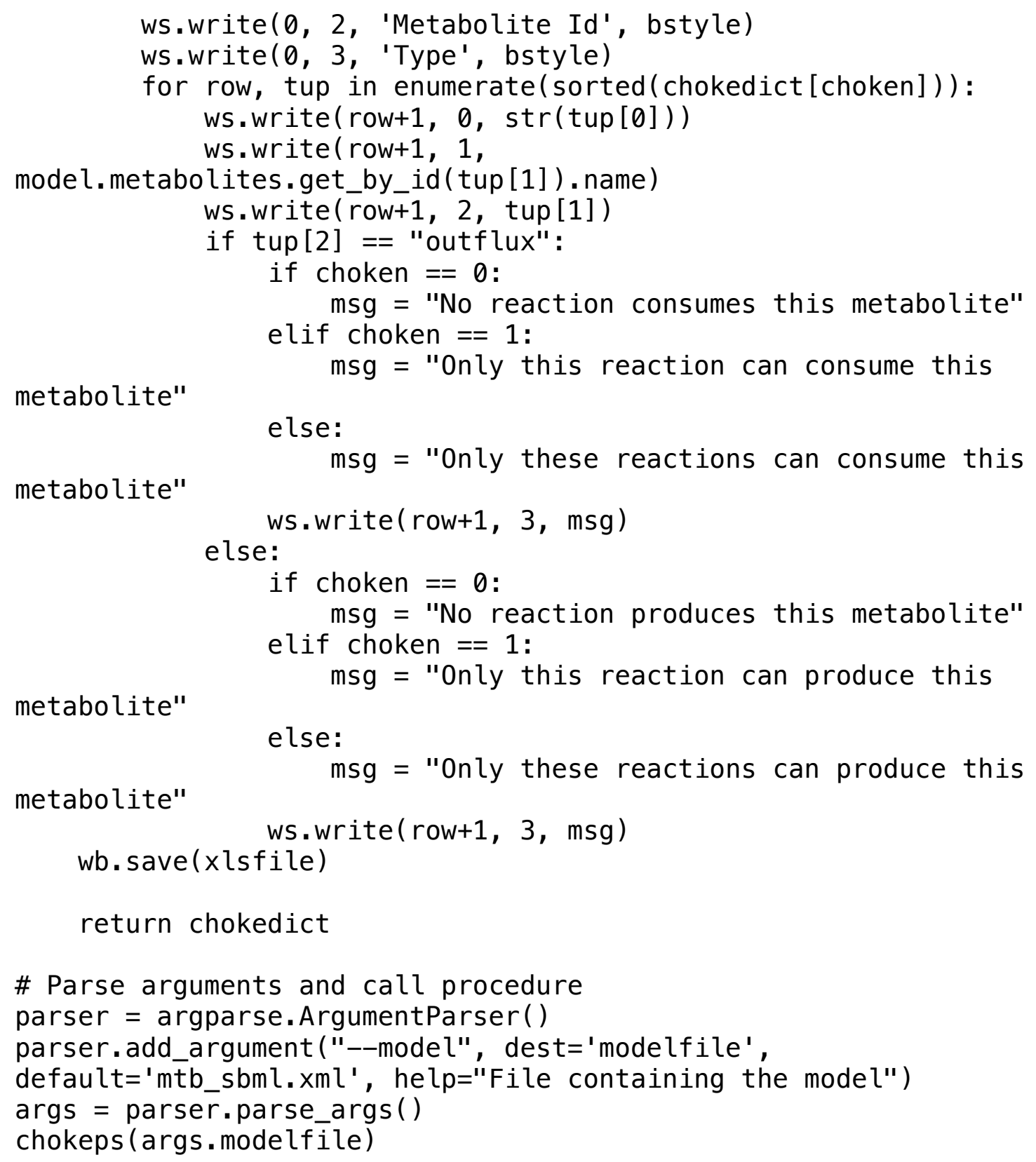

